\title{
Color preferences in infants and adults are different
}

\author{
Chloe Taylor • Karen Schloss • Stephen E. Palmer • \\ Anna Franklin
}

Published online: 23 February 2013

(C) Psychonomic Society, Inc. 2013

\begin{abstract}
Adults commonly prefer blues most and greenish yellows least, but these hue preferences interact with lightness and saturation (e.g., dark yellow is particularly disliked: Palmer \& Schloss (Proceedings of the National Academy of Sciences 107:8877-8882, 2010)). Here, we tested for a similar hue-by-lightness interaction in infant looking preferences, to determine whether adult preferences are evident early in life. We measured looking times for both infants and adults in the same paired-comparison task using all possible pairs of eight colors: four hues (red/yellow/green/blue) at two lightness levels (dark/light). The adult looking data were strikingly similar to other adults' explicit preference responses, indicating for the first time that adults look longer at colors that they like. Infants showed a significant hue-by-lightness interaction, but it was quite different from the adult pattern. In particular, infants had a stronger looking preference for dark yellow and a weaker preference for light blue than did adults. The findings are discussed in relation to theories on the origins of color preference.
\end{abstract}

Keywords Cognitive development - Visual perception . Aesthetics · Color

Prior research on color preference has established that adults commonly like some colors more than others-for instance, blue more than yellow (e.g., Eysenck, 1941; Guilford \& Smith, 1959; Hurlbert \& Ling, 2007; Palmer \& Schloss,

\section{Taylor}

University of Surrey, Guildford, Surrey, UK

K. Schloss $\cdot$ S. E. Palmer

University of California, Berkeley, CA, USA

A. Franklin $(\bowtie)$

School of Psychology, University of Sussex,

Brighton BN1 9RH, UK

e-mail: anna.franklin@sussex.ac.uk
2010). Several theories have recently been proposed about what governs these preferences (Hurlbert \& Ling, 2007; Ou, Luo, Woodcock, \& Wright, 2004; Palmer \& Schloss, 2010). The ecological valence theory (EVT: Palmer \& Schloss, 2010), for example, proposes that color preferences arise from affective responses to color-associated objects: People like/dislike colors to the degree that they like/dislike the objects that are characteristically associated with that color. In support, Palmer and Schloss found that $80 \%$ of the variance in American preferences for 32 colors could be accounted for by the average valence ratings of the objects associated with these colors. For example, strongly disliked dark greenish yellow was associated with strongly disliked entities such as dirty water, bile, and rotten food, whereas strongly liked saturated blue was associated with strongly liked entities such as clear sky and clean water.

EVT suggests that the association between object valences and color preferences could be learned during one's lifetime, being influenced by the objects that an individual experiences and his or her affective response to those objects. However, EVT also proposes that an innate, adaptive function of color preferences could draw us toward objects that are evolutionarily beneficial (e.g., clean water), and away from those that are not (e.g., rotten food). Previous research on infant color preferences has been consistent with the innateness hypothesis, in that young infants preferentially look most at blue and least at yellow-green or yellow (e.g., Adams, 1987; Bornstein, 1975; Franklin, Bevis, Ling, \& Hurlbert, 2010; Franklin et al., 2008; Teller, Civan, \& Bronson-Castain, 2004; Zemach, Chang, \& Teller, 2007). The similarity between infant hue preference curves in these studies and those in their adult study led Palmer and Schloss (2010) to suggest that at least some aspects of adult color preference may be innate.

Several issues complicate this comparison, however. First, Palmer and Schloss (2010) found that adult hue preferences interact with saturation and lightness: For instance, yellow was strongly disliked relative to other hues among 
"dark" colors, but not among "saturated" or "light" colors. The color preferences of infants and adults thus cannot easily be compared unless the stimuli are of the same lightness and saturation for both. The one previous study meeting this criterion did find similar preference curves for infant looking times and adult preference judgments when the stimuli were highly saturated monochromatic lights (Bornstein, 1975), but only one lightness level was tested. This result thus does not address the important question of whether infants show the same interaction between hue and lightness as adults that was reported by Palmer and Schloss.

Second, other studies of infant color preferences have reported patterns of infant color preference that match adult hue preferences less well. For example, Adams (1987) found that 3-month-old infants actually looked longer at yellow than blue. This discrepant observation, as well as the generally inconsistent pattern of hue preferences in previous infant studies, may also have been due to interactions of hue with saturation and/or lightness. Zemach et al. (2007) found a fairly consistent pattern of hue preference at different saturation levels, but they only tested one very dark lightness level $\left(4.5 \mathrm{~cd} / \mathrm{m}^{2}\right)$. The uncontrolled variations in lightness levels in previous research thus make it difficult to infer either whether infant hue preferences are constant throughout color space or how well they match adult preferences.

Third, adults' color preferences are typically measured by ratings or forced choices between pairs of alternatives, whereas infants' are measured in a preferential-looking paradigm. It is therefore completely unknown how adult and infant preferences would compare using the same behavioral tests.

In the present study, we investigated these issues by measuring infant and adult preferences using the same preferential-looking measure for the same four hues (red/yellow/green/blue) at two different lightness levels (light/dark) that were matched for chromatic contrast to the gray background, allowing for a direct comparison between the hue-by-lightness interactions for infant and adult color preferences. In Experiment 1, we investigated infants' preferential-looking behavior for all possible pairs of these eight colors. In Experiment 2, we considered adults' preferences for the same colors, using both explicit measures of color preference and a preferential-looking measure. The results will provide greater clarity as to whether the elements of adult color preference can be found in infants' responses to color.

\section{Experiment 1: Infant color preferences at 4-6 months}

In Experiment 1, we assessed color preferences in 4- to 6month-old infants by measuring looking times with an eyetracker (e.g., Franklin et al., 2010) for all possible pairs of eight colors: Dark and light versions of red, yellow, green, and blue. If we were to find an interaction between hue and lightness, it could explain the inconsistent results from previous infant studies.

Method

\section{Participants}

We tested thirty 4- to 6-month-old, full-term, English infants (19 male, 11 female; $M_{\text {age }}=22.85$ weeks, $S D=2.71$ ). Six other infants were excluded because general fussiness prevented them from completing the experiment.

\section{Stimuli and design}

The colors included four hues (red/yellow/green/blue) at two lightnesses (light/dark), with constant lightness and saturation (CIELUV) within a lightness level and constant chroma (equal chromatic distances to the gray background) across all colors (Table 1$)$. They were presented as circles $\left(8.39^{\circ}\right.$ diameter) on a gray background $\left(Y=16.52 \mathrm{~cd} / \mathrm{m}^{2}, x=.31\right.$, $y=.33$ ), to the left and right of central fixation (inner edge $6.27^{\circ}$ from fixation). Each color was paired with every other color, and the pairs were left/right balanced. The 56 trials were presented in random order.

Table 1 Chromaticity coordinates (CIE $x, y, 1931)$ for the stimuli used in Experiment 1 (denoted with a ${ }^{*}$ ) and Experiment 2 (all stimuli)

\begin{tabular}{|c|c|c|}
\hline Hue & $x$ & $y$ \\
\hline Light yellow ${ }^{*}$ & .36 & .39 \\
\hline Light orange & .37 & .36 \\
\hline Light red ${ }^{*}$ & .37 & .33 \\
\hline Light purple & .30 & .27 \\
\hline Light blue $^{*}$ & .26 & .29 \\
\hline Light cyan & .26 & .34 \\
\hline Light green* & .29 & .38 \\
\hline Light chartreuse & .34 & .40 \\
\hline Dark yellow ${ }^{*}$ & .41 & .44 \\
\hline Dark orange & .42 & .38 \\
\hline Dark red* & .40 & .33 \\
\hline Dark purple & .29 & .24 \\
\hline Dark blue ${ }^{*}$ & .23 & .26 \\
\hline Dark cyan & .22 & .35 \\
\hline Dark green* & .28 & .43 \\
\hline Dark chartreuse & .37 & .46 \\
\hline
\end{tabular}

L* was constant at $71.6\left(Y=27.56 \mathrm{~cd} / \mathrm{m}^{2}\right)$ for the light cut, and at $41.22\left(Y=7.68 \mathrm{~cd} / \mathrm{m}^{2}\right)$ for the dark cut, and was calculated using the white point of the monitor $\left(Y=64 \mathrm{~cd} / \mathrm{m}^{2}, x=.31, y=.33\right)$ as the reference white. Saturation (CIELUV) was constant at 0.63 for the light cut and 1.09 for the dark cut. The chroma (CIELUV) was 35 for all stimuli 


\section{Setup and procedure}

Infants sat in a car seat, $59 \mathrm{~cm}$ from a calibrated 21-in. CRT monitor (Sony Trinitron GDM-F520). A cartoon with music played while the experimenter focused the eyetracker (ASL 504 Pan/Tilt) on the infants' eyes and adjusted the pupil and corneal reflection thresholds. The infants' eye movements were calibrated by recording corneal reflections and pupil signals when a black-and-white "attention getter" that loomed synchronously with beeping sounds was presented at top left and bottom right monitor locations. Calibration accuracy was then tested with the same "attention getter" at five random points on the monitor. If the crosshairs indicating that the points of gaze were centered on the "attention getter" for all five points, calibration was judged to be accurate. Otherwise, the calibration procedure was repeated. The infant then completed the 56 experimental trials. Before each trial, the "attention getter" was shown at the fixation point. Once the infant's gaze was centrally fixated, the experimenter began the trial. If the infant's gaze wandered from the screen or their attention appeared to diminish, black-and-white cartoon animals that moved with synchronized sounds were played until the infant's gaze was again fixated on the screen, after which the next trial began.

\section{Results and discussion}

We calculated the total time that each infant spent looking directly at each color, and discarded all time spent looking elsewhere (Table 2). We found no overall preference among the four hues $\left[F(3,87)=1.84, p=.15, \eta_{\mathrm{p}}{ }^{2}=.06\right]$ or the two lightness levels $\left[F(1,29)=0.94, p=.34, \eta_{\mathrm{p}}{ }^{2}=.03\right]$. However, a significant interaction did emerge between hue and lightness $\left[F(3,87)=4.92, p<.005, \eta_{\mathrm{p}}{ }^{2}=.15\right]$, indicating that infant hue preferences do indeed differ across lightness levels. These results showed that infants' hue preferences are not consistent throughout color space. Among the light colors, infant looking times were longest for red and shortest for blue. Among the dark colors, they were longest for yellow and shortest for green.

We next compared looking biases for each color, $c$, to chance by converting total looking times for each individual, $i$, into relative looking-probability measures, $P_{c, i}=T_{c, i} / T_{i}$, where $T_{c, i}$ is the total time that an individual $i$ spent looking

Table 2 Average total looking times in seconds ( $S D \mathrm{~s}$ in parentheses) at the four hues, for light and dark stimulus cuts by 304 - to 6-monthold infants

\begin{tabular}{lllll}
\hline & Red & Yellow & Green & Blue \\
\hline Light & $10.31(3.9)$ & $9.04(4.5)$ & $8.76(3.8)$ & $8.80(4.2)$ \\
Dark & $9.33(3.7)$ & $10.73(4.9)$ & $8.79(4.6)$ & $9.99(5.1)$ \\
\hline
\end{tabular}

at color $c$, and $T_{i}$ is the total time that individual $i$ spent looking at all colors. This measure removes from the data differences among individual infants in total looking times and specifies chance at $P_{c, i}=.125$. Values significantly greater than .125 indicate a bias toward looking at color $c$, whereas those lower than .125 indicate a bias away from looking at color $c$ (see Fig. 1). Two-tailed $t$ tests revealed that light red $[t(28)=2.26, p=.03, d=0.42]$ and dark yellow $[t(28)=2.15, p=.04, d=0.40]$ were fixated more likely than chance, whereas light blue $[t(28)=-2.27, p=.03, d=0.42]$ and dark green $[t(28)=-2.75, p=.01, d=0.51]$ were fixated less likely than chance. For both light and dark colors, the highest and lowest preferences were significantly different from chance. This finding might explain the differences among previous infant studies (Adams, 1987; Bornstein, 1975; Franklin et al., 2010; Franklin et al., 2008; Teller et al., 2004; Zemach et al., 2007), because the lightnesses (and saturations) of colors were different in each study.

\section{Experiment 2: Adult color preferences}

The results of Experiment 1 provide little support for the notion that adult preferences arise from an "innate" bias toward blue and/or away from dark yellow. In fact, infants look significantly longer at dark yellow than chance, whereas Palmer and Schloss's (2010) previous results showed that adults rate that color as the least preferred among their 32 colors. It is possible that such differences arise from methodological differences between explicit preference ratings and looking-time measures, however. We avoided this objection in Experiment 2 by measuring adults' preferences for the same colors using the same looking-time measures that we had used in Experiment 1 with infants.

In addition to the eight colors from Experiment 1, we included light and dark versions of four intermediate hues (orange/chartreuse/cyan/purple) in order to provide a more detailed hue preference curve analogous to that of Palmer and Schloss (2010). We also tested for possible differences between looking-time measures and the more typical explicit measures of preference ratings and forced choice probabilities, to establish whether adults look more at colors that they like and less at colors that they dislike.

Method

\section{Participants}

We recruited 123 participants from the University of Surrey (61 males, 62 females; $M_{\text {age }}=20$ years, $S D_{\text {age }}=3.00$ ). All had normal color vision, as indicated by performance on the City Colour Vision test (Fletcher, 1980). 
Fig. 1 Infants' mean $( \pm 1 S E)$ relative probabilities of looking at the four hues, for light and dark cuts. The line at .125 on the $y$-axis indicates no looking bias

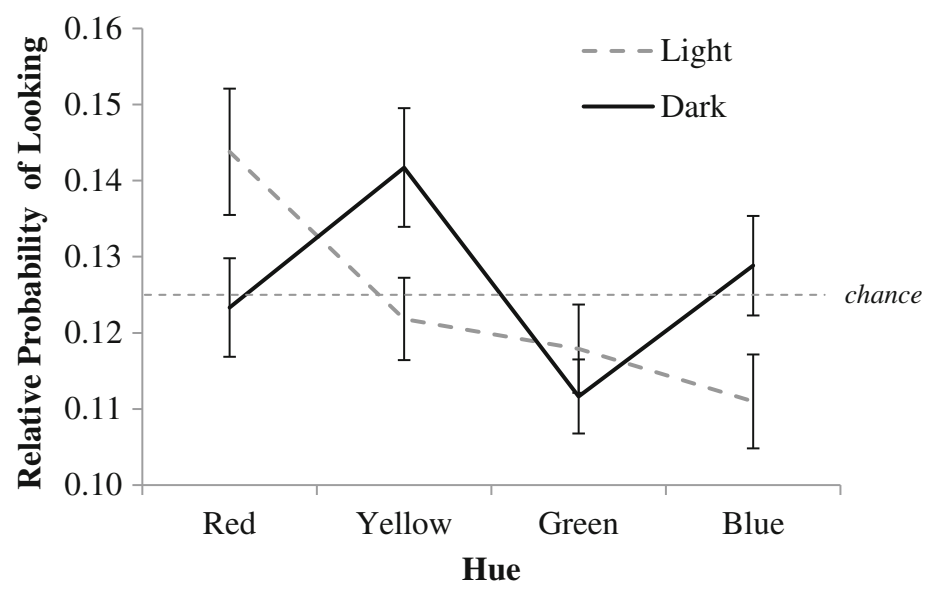

\section{Stimuli}

The colors and background were identical to those of Experiment 1, with the addition of four "intermediate hues" (orange/chartreuse/cyan/purple) that were similar to hues used by Palmer and Schloss (2010; see our Table 1). The size and location of the stimuli for the preferential-looking and preferential-choice tasks were identical to those aspects of the stimuli in Experiment 1. For the preference-rating task, the stimuli were presented centrally as single rectangular patches $\left(10.00^{\circ}\right.$ horizontal, $9.34^{\circ}$ vertical $)$.

\section{Design and procedure}

Participants were randomly assigned to one of the three following tasks.

Preferential-looking task This procedure was identical to that of Experiment 1, except for using a 9-point calibration procedure. A group of 40 participants were simply told to look at the colors, with no mention of preference. Four blocks (of 56 trials each) differed only in the subsets of colors paired: (a) dark and light red, yellow, green, and blue, just as in Experiment 1; (b) dark and light orange, chartreuse, cyan, and purple; (c) all eight light colors; and (d) all eight dark colors. The order of the blocks was randomized across participants.

Preferential-choice task This task was similar to the preferential-looking task, except that 40 different participants were explicitly asked to click on their preferred color in each pair quickly and without considering any use for the colors. The gray background was presented between trials with a small achromatic central fixation patch. Participants pressed a key to begin each trial. All other aspects were identical to those of the preferential-looking task.

Preference-rating task Each color was presented individually on the same gray background. A group of 43 different participants rated their preferences for each color by sliding the cursor along a line-mark response scale and clicking to record their responses. The 400pixel scale was positioned below the color patch, with the left end labeled not at all and the right end labeled very much. The cursor was always initialized at the central neutral point, indicated by a vertical tick mark. Participants were instructed to respond quickly without thinking about any possible use of the colors. The colors were presented in random order, and the gray background was displayed between trials until participants pressed a key to start the next trial.

Results and discussion

The adult preferential looking-time data were first analyzed for the Experiment 1 colors (light and dark red, yellow, green, and blue) in the same way as for the infant data (Table 3 ). We found main effects of hue $[F(3,59)=29.86$, $\left.p<.001, \eta_{\mathrm{p}}{ }^{2}=.34\right]$, with bluer colors being preferred, and lightness $\left[F(1,59)=13.36, p=.001, \eta_{\mathrm{p}}{ }^{2}=.19\right]$, with lighter colors being preferred, and a significant hue-by-lightness interaction $\left[F(3,177)=3.80, p<.05, \eta_{\mathrm{p}}{ }^{2}=.06\right]$ due to the larger reduction in looking times for dark yellow.

These data were then converted to relative probabilities of looking at each color, as described in Experiment 1 (Fig. 2). Tests against chance $\left(P_{c, i}=.125\right)$ revealed significant biases toward looking at light red, light blue, and light green more than chance $[t \mathrm{~s}(59)=2.51,6.16$, and $3.03 ; p \mathrm{~s}=.015, .001$, and $.004 ; d \mathrm{~s}=0.31,0.79$, and 0.39 , respectively] and looking at dark yellow less than chance $[t(59)=-8.45, p<.001]$. The

Table 3 Total looking times in seconds ( $S D$ s in parentheses) at the four hues, for light and dark cuts by adults

\begin{tabular}{lllll}
\hline & Red & Yellow & Green & Blue \\
\hline Light & $17.54(5.5)$ & $14.96(3.7)$ & $17.27(4.0)$ & $18.86(3.9)$ \\
Dark & $14.56(4.8)$ & $10.55(4.9)$ & $15.01(5.1)$ & $17.15(4.5)$ \\
\hline
\end{tabular}


Fig. 2 Adults' mean $( \pm 1 S E)$ at the four hues, for light and dark cuts. The line at .125 on the $y$-axis indicates no looking bias relative probabilities of looking

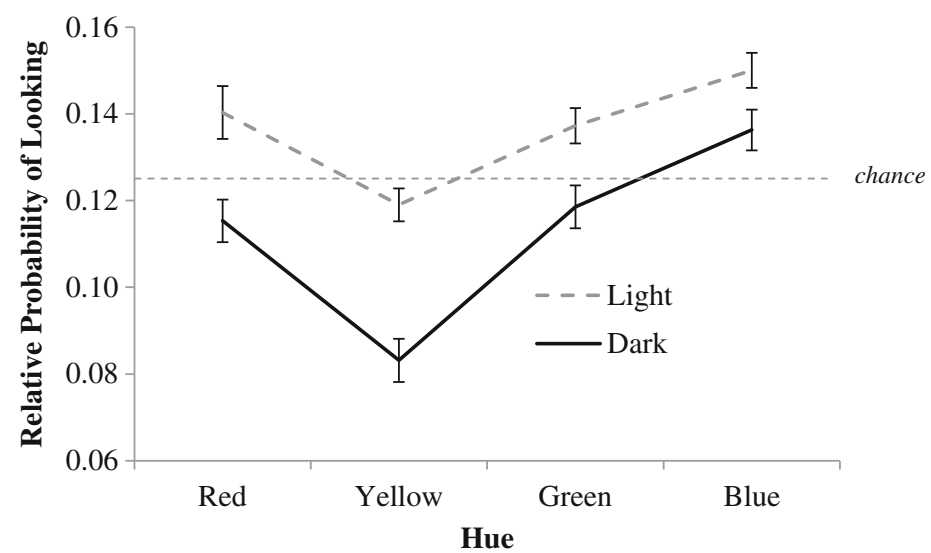

\section{General discussion}

not significantly correlated $(r=-.39, p=.33)$.

Preferential looking for the adults in Experiment 2 (Fig. 2) differed in a number of ways from that for infants in Experiment 1 (Fig. 1). In particular, adults looked significantly less than chance at dark yellow, whereas infants looked significantly more than chance at this color. The adults also looked significantly more than chance at light blue and light green, whereas infants did not. The primary similarity in preferential looking between infants and adults was that both looked significantly longer at light red (pink) than would be expected by chance.

To determine how such looking probabilities relate to more standard measures of color preference in adults, we compared them with two-alternative forced choice probabilities and explicit preference ratings. All three measures were first linearly transformed to range from -1 to +1 (Fig. 3). ${ }^{1}$ For the looking and forced choice measures, a probability of 0 mapped to -1 , a probability of .125 mapped to 0 (i.e., chance, or no preference), and a probability of .25 mapped to +1 , as this was the highest probability with which a color could be looked at or chosen. For the preference ratings, -400 pixels mapped to -1 and +400 pixels to +1 . Looking probabilities were highly correlated with the choice probabilities $(r=.94, p<.001)$ and with preferential ratings $(r=.91, p<.001)$, indicating that people spend more time looking at colors that they like. Because the adults were simply told to look at the colors, with no mention made of preference at all, the similarity of the looking behavior to the explicit preference ratings is striking. To the best of our knowledge, this is the first demonstration that adults look longer at colors that they like.

\footnotetext{
${ }^{1}$ The three measures were transformed to the same -1 to +1 scale using the following formulae: transformed $P_{c, i}=\left(P_{c, i}-.125\right) / .125$; transformed two-alternative forced choice (2AFC) probabilities = $(2 \mathrm{AFC}$ probability -7$) / 7$; transformed preference rating $=$ preference rating/400.
}

The results of two experiments established clear differences between the preferential-looking behavior of 4- to 6-monthold infants and adults for colors that differed in hue and lightness. Infants looked at dark yellow longer than chance, whereas adults looked at it less than chance. Also, adults looked longer at light blue and light green than would be expected by chance, yet infants did not. Therefore, at least for the colors that we tested, there was evidence of neither an "innate" looking preference for blue hues nor an "innate" aversion to dark yellow.

The present pattern of interaction between hue and lightness may explain inconsistencies in the patterns of infant hue preference reported in previous studies. For example, several results have indicated a strong looking preference for red in young infants (Adams, 1987; Bornstein, 1975; Franklin et al., 2010), whereas others have found a relatively reduced preference for red (Teller et al., 2004; Zemach et al., 2007). Such differences could well be due to differences in the lightness (and/or saturation) of the reds across studies. Previous research has also shown an infant preference for blue when the stimuli are highly saturated (e.g., Bornstein, 1975; Franklin et al., 2008; Zemach et al., 2007), but the present results showed that for less saturated colors, this preference for blue diminishes, especially for light blue. One possible explanation is that the strength of the infant preference for blue relative to other hues could be modulated by the strength of "blue-yellow" tritan discrimination at different saturation levels, as there is some suggestion that the tritan response can be diminished in infants at lower saturations (e.g., Teller, 1998). Further research will be needed to investigate these issues and to understand the nature of infant color preferences in three-dimensional color space.

The primary similarity that we found between infant and adult preferential-looking behavior was that both looked longer at light red (pink) than would be expected by chance. This similarity may be coincidental, but it could also arise 


\section{LIGHT CUT}

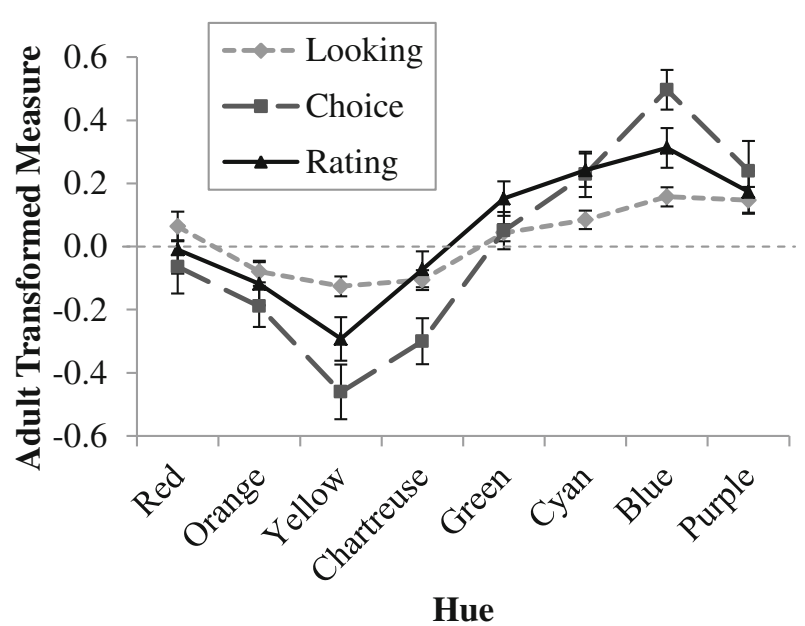

\section{DARK CUT}

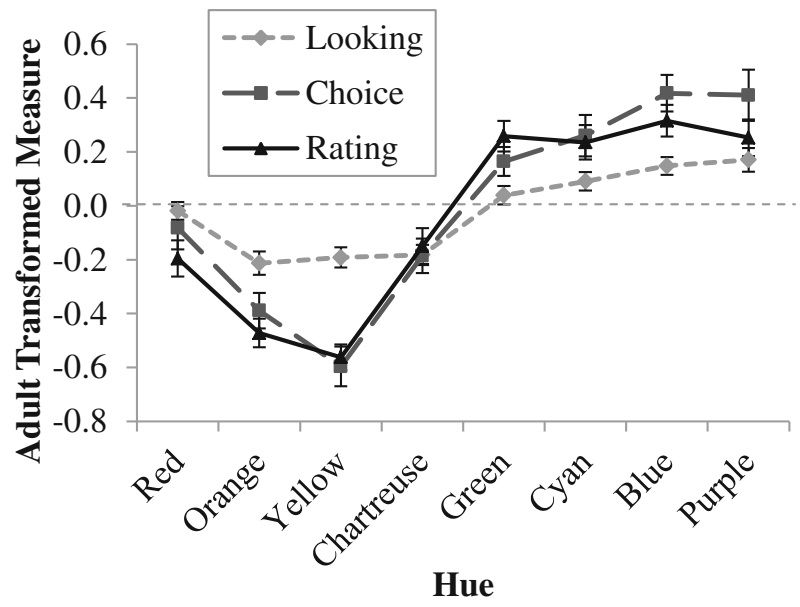

Fig. 3 The curves for looking probabilities, choice probabilities, and preference ratings when all have been transformed to the same -1 to +1 scale, for the light cut (top) and the dark cut (bottom). Error bars indicate $\pm 1 S E$

from the privileged status of desaturated reddish colors in human perception. Lindsey et al. (2010) found an advantage for desaturated reddish targets in chromatic search, with minimum search times for colors located near the chromatic locus of skin and lips. The light red in the present study closely corresponded to the chromatic locus of lips (the CIELAB hue angle of the light red stimulus was $23.61^{\circ}$, and the chromatic locus of lips is around $30^{\circ}$; Gozalo-Dias, Lindsey, Johnston, \& Wee, 2007). Therefore, one very tentative suggestion is that infants looked longer than chance at the light reddish hue because it is evolutionarily advantageous for infants to be drawn to the color of lips, in that attention to the mouth is important for detecting emotional expressions and language learning (even if young infants preferentially fixate the eyes over the mouth; e.g., Haith, Bergman, \& Moore, 1977; Maurer \& Salapatek, 1976; but see also Hunnius \& Geuze, 2004). In this sense, some aspects of infants' preferential looking at colors could well be ecological. This could be tested directly by measuring preferences for colors associated with other entities important for infants' development or survival, such as nipples.

The present data also show that adults look longer at colors that they like than at colors that they dislike. This novel finding raises the question of whether color preference affects other aspects of color perception, such as chromatic search: For example, do adults detect colors that they like more quickly than those that they dislike? Such an effect would be consistent with the EVT, as it could serve to allocate attention more quickly, and sustain attention longer, for liked than for disliked objects. Although much research has investigated adult color preferences and the factors that govern these preferences, little is known about how these preferences impact perception and cognition. We suggest that this could be a worthwhile avenue for further research.

Although increased/decreased looking appears to indicate increased/decreased liking in adults, we caution against assuming that the same is true for infants. Theoretically, infants may look longer at the colors because of factors other than liking - for instance, complexity, salience, or novelty (Franklin, Gibbons, Chittenden, Taylor, \& Alvarez, 2012) - and looking may become associated with liking later in development. One current challenge in infant research is how to measure whether a young infant has an emotional response or "likes" something (Quinn et al., 2011). One possibility is to measure infants' affect via behavioral responses to stimuli, such as facial expressions (e.g., using an electromyographic measure). Studies of this kind may be helpful in providing greater clarity as to what colors infants actually "like." Nevertheless, the present data at least establish that adult color preferences are not closely related to infants' preferential looking at colors.

One potential account of the present data is that different responses to color among infants and adults could be explained by differences in their color vision. However, infants are known to be trichromatic by at least 3 months (Knoblauch, Vital-Durand, \& Barbur, 2001), and adult isoluminance is also thought to be equivalent to that of infants (Pereverzeva, Chien, Palmer, \& Teller, 2002). As we discussed earlier, it is possible that infants' tritan response is diminished for colors at the present saturation levels (Teller, 1998), and this could potentially account for the different patterns of preference in infants and adults. However, previous research has established only a weak link between chromatic discrimination thresholds and preferential looking at colors in infants at 12 weeks of age (Zemach et al., 2007).

An alternative possibility, which is suggested by the EVT, is that differences in infant and adult preferential looking at colors can be explained by differences in color- 
object associations and object valences for infants and adults. Adult color preferences appear to be related to the color-object associations that adults make (Palmer \& Schloss, 2010; Taylor \& Franklin, 2012), at least for some cultures (Taylor, Clifford \& Franklin, 2012). Infants may be free of these associations, or they may form different associations and/or have different object valences. Research suggests that infants can form color-object associations by as young as 6 months (Kimura et al., 2010), which raises the interesting possibility that colors have associated object valences in infancy as well. Further investigation of colorobject associations and their relationship to color preferences in infancy will be needed to investigate the plausibility of such explanations.

In conclusion, the present results show little evidence that adult color preferences are evident in infants' preferentiallooking responses to color. This is true despite the fact that adult preferential-looking behavior is closely related to adult responses in explicit color preference tasks. The present results also show, for the first time, that infant hue preferences interact with lightness, thus providing a potential explanation for the discrepancies in hue preferences across previous infant studies (Adams, 1987; Bornstein, 1975; Franklin et al., 2010; Franklin et al., 2008; Teller et al., 2004; Zemach et al., 2007). Further research will be needed to determine how infants' preferential-looking relates to developing color vision, if at all, and to establish whether infant color preferences relate to color-object associations, as is suggested by the EVT (Palmer \& Schloss, 2010).

Author note We are grateful to Virginia Smith and Matthew Bosworth for assistance with the preliminary adult data collection. This research was supported in part by a PhD studentship from the University of Surrey to C.T., the National Science Foundation (Grant Nos. 0745820 and 1059088), and a Google Gift to S.E.P. Any opinions, findings, and conclusions or recommendations expressed in this material are those of the author(s) and do not necessarily reflect the views of the National Science Foundation or Google.

\section{References}

Adams, R. J. (1987). An evaluation of color preference in early infancy. Infant Behavior \& Development, 10, 143-150.

Bornstein, M. H. (1975). Qualities of color vision in infancy. Journal of Experimental Child Psychology, 19, 401-419.

Eysenck, H. J. (1941). A critical and experimental study of colour preferences. The American Journal of Psychology, 54, 385-394.

Fletcher, R. (1980). The City University Colour Vision Test (2nd Edition). London: Keller.

Franklin, A., Bevis, L., Ling, Y., \& Hurlbert, A. (2010). Biological components of colour preference in infancy. Developmental Science, 13, 346-354.
Franklin, A., Gibbons, E., Chittenden, K., Taylor, C., \& Alvarez, J. (2012). Infant color preference for red is not selectively context specific. Emotion, 12, 1155-1160.

Franklin, A., Pitchford, N., Hart, L., Davies, I. R. L., Clausse, S., \& Jennings, S. (2008). Salience of primary and secondary colours in infancy. British Journal of Developmental Psychology, 26, 471483. doi:10.1348/026151007X256672

Gozalo-Dias, D. J., Lindsey, D. T., Johnston, W. M., \& Wee, A. G. (2007). Measurement of color for craniofacial structures using a 45/0-degree optical configuration. Journal of Prosthetic Dentistry, 97, 45-53. doi:10.1016/j.prosdent.2006.10.013

Guilford, J. P., \& Smith, P. C. (1959). A system of color-preferences. The American Journal of Psychology, 72, 487-502.

Haith, M. M., Bergman, T., \& Moore, M. J. (1977). Eye contact and face scanning in early infancy. Science, 198, 853-855.

Hunnius, S., \& Geuze, R. H. (2004). Developmental changes in visual scanning of dynamic faces and abstract stimuli in infants: A longitudinal study. Infancy, 6, 231-255.

Hurlbert, A. C., \& Ling, Y. (2007). Biological components of sex differences in color preference. Current Biology, 17, R623R625. doi:10.1016/j.cub.2007.06.022

Kimura, A., Wada, Y., Yang, J., Otsuka, Y., Dan, I., Masuda, T.,... Yamaguchi, M. K. (2010). Infants' recognition of objects using canonical color. Journal of Experimental Child Psychology, 54, 363-368.

Knoblauch, K., Vital-Durand, F., \& Barbur, J. L. (2001). Variation of chromatic sensitivity across the life span. Vision Research, 41, $23-36$.

Lindsey, D. T., Brown, A. M., Reijnen, E., Rich, A. N., Kuzmova, Y. I., \& Wolfe, J. M. (2010). Color channels, not color appearance or color categories, guide visual search for desaturated color targets. Psychological Science, 21, 1208-1214. doi:10.1177/ 0956797610379861

Maurer, D., \& Salapatek, P. (1976). Developmental changes in the scanning of faces by young infants. Child Development, 47, 523-527.

Ou, L.-C., Luo, M. R., Woodcock, A., \& Wright, A. (2004). A study of colour emotion and colour preference: Part III. Colour preference modeling. Color Research and Application, 29, 381-389.

Palmer, S. E., \& Schloss, K. B. (2010). An ecological valence theory of human color preference. Proceedings of the National Academy of Sciences, 107, 8877-8882. doi:10.1073/pnas.0906172107

Pereverzeva, M., Chien, S. H., Palmer, J., \& Teller, D. Y. (2002). Infant photometry: Are mean adult isoluminance values a sufficient approximation to individual infant values? Vision Research, 42, 1639-1649.

Quinn, P. C., Anzures, G., Izard, C. E., Lee, K., Pascalis, O., Slater, A. M., \& Tanaka, J. W. (2011). Looking across domains to understand infant representation of emotion. Emotion Review, 3, 1-10.

Taylor, C., Clifford, A., \& Franklin, A. (2012). Color preferences are not universal. Journal of Experimental Psychology: General. doi:10.1037/a0030273

Taylor, C., \& Franklin, A. (2012). The relationship between color preference and color-object associations: Further investigation of ecological valence theory. Psychonomic Bulletin \& Review, 19, 190-197.

Teller, D. Y. (1998). Spatial and temporal aspects of infant color vision. Vision Research, 38, 3275-3282.

Teller, D. Y., Civan, A., \& Bronson-Castain, K. (2004). Infants' spontaneous color preferences are not due to adult-like brightness variations. Visual Neuroscience, 21, 397-401.

Zemach, I., Chang, S., \& Teller, D. Y. (2007). Infant color vision: Prediction of infants' spontaneous color preferences. Vision Research, 47, 1368-1381. 